\title{
The Class of Simple Cube-Curves Whose MLPs Cannot Have Vertices at Grid Points
}

\author{
Fajie Li and Reinhard Klette \\ CITR, University of Auckland, Tamaki Campus, \\ Building 731, Auckland, New Zealand
}

\begin{abstract}
We consider simple cube-curves in the orthogonal 3D grid of cells. The union of all cells contained in such a curve (also called the tube of this curve) is a polyhedrally bounded set. The curve's length is defined to be that of the minimum-length polygonal curve (MLP) fully contained and complete in the tube of the curve. So far only one general algorithm called rubber-band algorithm was known for the approximative calculation of such a MLP. There is an open problem which is related to the design of algorithms for calculation a 3D MLP of a cube-curve: Is there a simple cube-curve such that none of the vertices of its 3D MLP is a grid vertex? This paper constructs an example of such a simple cube-curve. We also characterize this class of cube-curves.
\end{abstract}

\section{Introduction}

The analysis of cube-curves is related to 3D image data analysis. A cube-curve is, for example, the result of a digitization process which maps a curve-like object into a union $S$ of face-connected closed cubes. The length of a simple cubecurve in 3D Euclidean space is based on the calculation of the minimal length polygonal curve (MLP) in a polyhedrally bounded compact set [3, 4.

The computation of the length of a simple cube-curve in 3D Euclidean space was a subject in [5]. But the method may fail for specific curves. [1] presents an algorithm (rubber-band algorithm) for computing the approximating MLP in $\mathrm{S}$ with measured time complexity in $O(n)$, where $n$ is the number of grid cubes of the given cube-curve.

The difficulty of the computation of the MLP in 3D may be illustrated by the fact that the Euclidean shortest path problem (i.e., find a shortest obstacleavoiding path from source point to target point, for a given finite collection of polyhedral obstacles in 3D space and a given source and a target point) is known to be NP-complete [7]. However, there are some algorithms solving the approximate Euclidean shortest path problem in 3D with polynomial-time, see [8]. The Rubber-band algorithm is not yet proved to be always convergent to the correct $3 \mathrm{D}-\mathrm{MLP}$.

Recently, 6] developed of an algorithm for calculation of the correct MLP (with proof) for a special class cube-curves. The main idea is to discompose the cube-curve into some arcs by finding some "end angles" (see Definition 4 below). 
There is an open problem (see [2-page 406]) which is related to designing algorithms for the calculation of the 3D MLP of a cube-curve: It there a simple cube-curve such that none of the vertices of its 3D MLP is a grid vertex? This paper constructs an example of such a simple cube-curve, and generalizes this by characterizing the class of all of those cube-curves. Furthermore it is true that these cube-curves do not have any end angle; and this means that we cannot use the MLP algorithm proposed in [6] which is provable correct. This is the basic importance of the given result: we show the existence of cube-curves which require further algorithmic studies.

Following [1, a grid point $(i, j, k) \in \mathbb{Z}^{3}$ is assumed to be the center point of a grid cube with faces parallel to the coordinate planes, with edges of length 1 , and vertices as its corners. Cells are either cubes, faces, edges, or vertices. The intersection of two cells is either empty or a joint side of both cells. A cube-curve is an alternating sequence $g=\left(f_{0}, c_{0}, f_{1}, c_{1}, \ldots, f_{n}, c_{n}\right)$ of faces $f_{i}$ and cubes $c_{i}$, for $0 \leq i \leq n$, such that faces $f_{i}$ and $f_{i+1}$ are sides of cube $c_{i}$, for $0 \leq i \leq n$ and $f_{n+1}=f_{0}$. It is simple iff $n \geq 4$ and for any two cubes $c_{i}, c_{k} \in g$ with $|i-k| \geq 2$ $(\bmod n+1)$, if $c_{i} \bigcap c_{k} \neq \phi$ then either $|i-k|=2(\bmod n+1)$ and $c_{i} \bigcap c_{k}$ is an edge, or $|i-k| \geq 3(\bmod n+1)$ and $c_{i} \bigcap c_{k}$ is a vertex.

A tube $\mathbf{g}$ is the union of all cubes contained in a cube-curve $g$. A tube is a compact set in $\mathbb{R}^{3}$, its frontier defines a polyhedron, and it is homeomorphic with a torus in case of a simple cube-curve. A curve in $\mathbb{R}^{3}$ is complete in $\mathbf{g}$ iff it has a nonempty intersection with every cube contained in $g$. Following [3, 4, we define:

Definition 1. A minimum-length polygon (MLP) of a simple cube-curve $g$ is a shortest simple curve $P$ which is contained and complete in tube $\boldsymbol{g}$. The length of a simple cube-curve $g$ is defined to be the length $l(P)$ of an MLP $P$ of $g$.

It turns out that such a shortest simple curve $P$ is always a polygonal curve, and it is uniquely defined if the cube-curve is not only contained in a single layer of cubes of the 3D grid (see 3, 4]). If it is contained in one layer, then the MLP is uniquely defined up to a translation orthogonal to that layer. We speak about the MLP of a simple cube-curve.

A critical edge of a cube-curve $g$ is such a grid edge which is incident with exactly three different cubes contained in $g$. Figure 1 shows all the critical edges of a simple cube-curve.

Definition 2. If $e$ is a critical edge of $g$ and $l$ is a straight line such that $e \subset l$, then $l$ is called a critical line of $e$ in $g$ or critical line for short.

Definition 3. Let e be a critical edge of $g$. Let $P_{1}$ and $P_{2}$ be the two end points of e. If one of coordinates of $P_{1}$ is less than that of $P_{2}$, then $P_{1}$ is called the first end point of $e$ in $g$. Otherwise $P_{1}$ is called the second end point of $e$ in $g$.

Definition 4. Assume a simple cube-curve $g$ and a triple of consecutive critical edges $e_{1}, e_{2}$, and $e_{3}$ such that $e_{i} \perp e_{j}$, for all $i, j=1,2,3$ with $i \neq j$. If $e_{2}$ is parallel to the $x$-axis (y-axis, or $z$-axis) implies the $x$-coordinates (y-coordinates, 
or z-coordinates) of two vertices (i.e., end points) of $e_{1}$ and $e_{3}$ are equal, then we say that $e_{1}, e_{2}$ and $e_{3}$ form an end angle, and $g$ has an end angle, denoted by $\angle\left(e_{1}, e_{2}, e_{3}\right)$; otherwise we say that $e_{1}, e_{2}$ and $e_{3}$ form a middle angle, and $g$ has a middle angle.

Figure 1 shows a simple cube-curve which has 5 end angles $\angle\left(e_{21}, e_{0}, e_{1}\right)$, $\left.\angle\left(e_{4}, e_{5}, e_{6}\right), \angle\left(e_{6}, e_{7}, e_{8}\right), \angle\left(e_{14}, e_{15}, e_{16}\right)\right), \angle\left(e_{16}, e_{17}, e_{18}\right)$, and many middle angles (e.g., $\angle\left(e_{0}, e_{1}, e_{2}\right), \angle\left(e_{1}, e_{2}, e_{3}\right)$, or $\left.\angle\left(e_{2}, e_{3}, e_{4}\right)\right)$.

Definition 5. A simple cube-curve $g$ is called first class iff each critical edge of $g$ contains exactly one vertex of the MLP of $g$.

We can simply detect a simple cube-curve is first class or not by running rubber band algorithm: the curve is first class iff option $\left(O_{1}\right)$ (see [1]) does not occur.

This paper focuses on first-class simple cube-curves because the general simple cube-curves require further studies.

Definition 6. Let $S \subseteq \mathbb{R}^{3}$. The set $\{(x, y, 0): \exists z(z \in \mathbb{R} \wedge(x, y, z) \in S)\}$ is the $x y$-projection of $S$, or projection of $S$ for short. Analogously we define the $y z-$ or $x z$-projection of $S$.

Definition 7. If $e_{1}, e_{2}, \ldots, e_{m}$ are consecutive critical edges of a cube-curve $g$ and $e_{0} \perp e_{1}, e_{m} \perp e_{m+1}$, and $e_{i} \| e_{i+1}$, where $i$ equals $1,2, \ldots$, and $m-1$, $m \geq 2$, then $\left\{e_{1}, e_{2}, \ldots, e_{m}\right\}$ is a set of maximal parallel critical edges of $g$, and critical edge $e_{0}$ or $e_{m+1}$ is called adjacent to this set.

Figure 1 shows a simple cube-curve which has 2 maximal parallel critical edge sets: $\left\{e_{11}, e_{12}\right\}$ and $\left\{e_{18}, e_{19}, e_{20}, e_{21}\right\}$. The two adjacent critical edges of

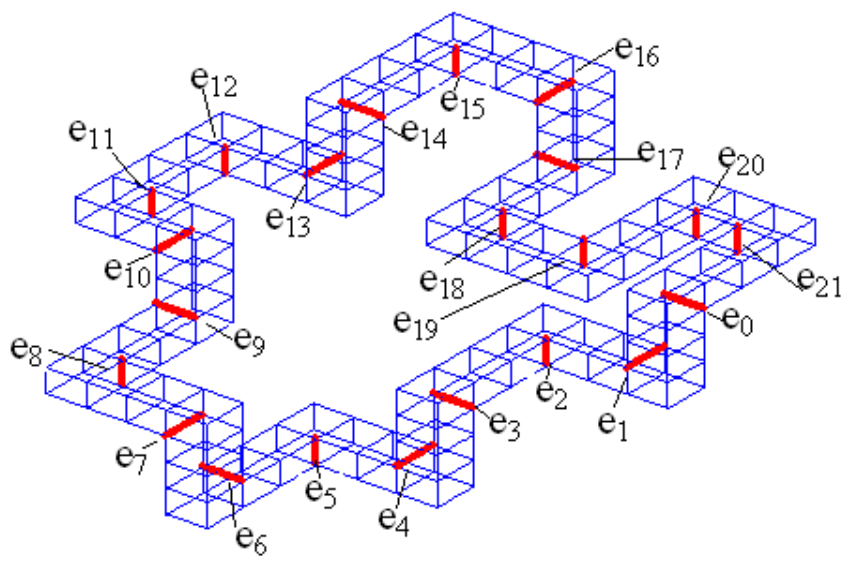

Fig. 1. Example of a first-class simple cube-curve which has middle and end angles 
$\left\{e_{11}, e_{12}\right\}$ are $e_{10}$ and $e_{13}$, they are on two different grid planes. The two adjacent critical edges of $\left\{e_{18}, e_{19}, e_{20}, e_{21}\right\}$ are $e_{17}$ and $e_{0}$, they are on two different grid planes as well.

The paper is organized as follows: Section 2 describes theoretical fundamentals for constructing our example. Section 3 presents the example. Section 4 gives the conclusions.

\section{Basics}

We provide mathematical fundamentals used for constructing a simple cubecurve such that none of the vertices of its 3D MLP is a grid vertex. We start with citing a basic theorem from [1]:

Theorem 1. Let $g$ be a simple cube-curve. Critical edges are the only possible locations of vertices of the MLP of $g$.

Let $d_{e}(p, q)$ be the Euclidean distance between points $p$ and $q$.

Let $e_{0}, e_{1}, e_{2}, \ldots, e_{m}$ and $e_{m+1}$ be $m+2$ consecutive critical edges in a simple cube-curve, and let $l_{0}, l_{1}, l_{2}, \ldots, l_{m}$ and $l_{m+1}$ be the corresponding critical lines. We express a point $p_{i}\left(t_{i}\right)=\left(x_{i}+k_{x_{i}} t_{i}, y_{i}+k_{y_{i}} t_{i}, z_{i}+k_{z_{i}} t_{i}\right)$ on $l_{i}$ in general form, with $t_{i} \in \mathbb{R}$, where $i$ equals $0,1, \ldots$, or $m+1$.

In the following, $p\left(t_{i}\right)$ will be denoted by $p_{i}$ for short, where $i$ equals $0,1, \ldots$, or $m+1$.

Lemma 1. If $e_{1} \perp e_{2}$, then $\frac{\partial d_{e}\left(p_{1}, p_{2}\right)}{\partial t_{2}}$ can be written as $\left(t_{2}-\alpha\right) \beta$, where $\beta>0$, and $\beta$ is a function of $t_{1}$ and $t_{2}, \alpha$ is 0 if $e_{1}$ and the first end point of $e_{2}$ are on the same grid plane, and $\alpha$ is 1 otherwise.

Proof. Without loss of generality, we can assume that $e_{2}$ is parallel to $z$-axis. In this case, the parallel projection (denoted by $g^{\prime}\left(e_{1}, e_{2}\right)$ ) of all of $g$ 's cubes, contained between $e_{1}$ and $e_{2}$, is illustrated in Figure2, where $A B$ is the projective image of $e_{1}$, and $C$ is that of one of the end points of $e_{2}$.

Case 1. $e_{1}$ and the first end point of $e_{2}$ are on the same grid plane. Let the two end points of $e_{2}$ be $(a, b, c)$ and $(a, b, c+1)$. Then the two end points of $e_{1}$ are

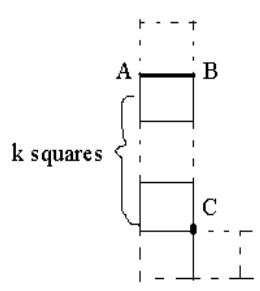

Fig. 2. Illustration of the proof of Lemma 1 
$(a-1, b+k, c)$ and $(a, b+k, c)$. Then the coordinates of $p_{1}$ and $p_{2}$ are $(a-1+$ $\left.t_{1}, b+k, c\right)$ and $\left(a, b, c+t_{2}\right)$ respectively, and $d_{e}\left(p_{1}, p_{2}\right)=\sqrt{\left(t_{1}-1\right)^{2}+k^{2}+t_{2}^{2}}$.

Therefore $\frac{\partial d_{e}\left(p_{1}, p_{2}\right)}{\partial t_{2}}=\frac{t_{2}}{\sqrt{\left(t_{1}-1\right)^{2}+k^{2}+t_{2}^{2}}}$. Let $\alpha=0$ and $\beta=\frac{1}{\sqrt{\left(t_{1}-1\right)^{2}+k^{2}+t_{2}^{2}}}$. This proves the lemma for Case 1.

Case 2. $e_{1}$ and the first end point of $e_{2}$ are on different grid planes (i.e., $e_{1}$ and the second end point of $e_{2}$ are on the same grid plane). Let the two end points of $e_{2}$ be $(a, b, c)$ and $(a, b, c+1)$. Then the two end points of $e_{1}$ are $(a-1, b+k, c+1)$ and $(a, b+k, c+1)$. Then the coordinates of $p_{1}$ and $p_{2}$ are $\left(a-1+t_{1}, b+k, c+1\right)$ and $\left(a, b, c+t_{2}\right)$ respectively, and $d_{e}\left(p_{1}, p_{2}\right)=\sqrt{\left(t_{1}-1\right)^{2}+k^{2}+\left(t_{2}-1\right)^{2}}$.

Therefore $\frac{\partial d_{e}\left(p_{1}, p_{2}\right)}{\partial t_{2}}=\frac{t_{2}-1}{\sqrt{\left(t_{1}-1\right)^{2}+k^{2}+\left(t_{2}-1\right)^{2}}}$. Let $\alpha=1$ and

$\beta=\frac{1}{\sqrt{\left(t_{1}-1\right)^{2}+k^{2}+\left(t_{2}-1\right)^{2}}}$. This proves the lemma for Case 2 .

Lemma 2. If $e_{1} \| e_{2}$, then $\frac{\partial d_{e}\left(p_{1}, p_{2}\right)}{\partial t_{2}}$ can be written as $\left(t_{2}-t_{1}\right) \beta$, where $\beta>0$, and $\beta$ is a function of $t_{1}$ and $t_{2}$

Proof. Without loss of generality, we can assume that $e_{2}$ is parallel to $z$-axis. In this case, the parallel projection (denoted by $g^{\prime}\left(e_{1}, e_{2}\right)$ ) of all of $g$ 's cubes contained between $e_{1}$ and $e_{2}$ is illustrated in Figure 3, where $A$ is the projective image of one of the end points of $e_{1}$, and $B$ is that of one of the end points of $e_{2}$.

Case 1. $e_{1}$ and $e_{2}$ are on the same grid plane. Let the two end points of $e_{2}$ be $(a, b, c)$ and $(a, b, c+1)$. Then the two end points of $e_{1}$ are $(a, b+k, c)$ and $(a, b+k, c+1)$. Then the coordinates of $p_{1}$ and $p_{2}$ are $\left(a, b+k, c+t_{1}\right)$ and $\left(a, b, c+t_{2}\right)$ respectively, and $d_{e}\left(p_{1}, p_{2}\right)=\sqrt{\left(t_{2}-t_{1}\right)^{2}+k^{2}}$.

Therefore $\frac{\partial d_{e}\left(p_{1}, p_{2}\right)}{\partial t_{2}}=\frac{t_{2}-t_{1}}{\sqrt{\left(t_{2}-t_{1}\right)^{2}+k^{2}}}$. Let $\beta=\frac{1}{\sqrt{\left(t_{2}-t_{1}\right)^{2}+k^{2}}}$. This proves the lemma for Case 1.

Case 2. $e_{1}$ and $e_{2}$ are on different grid planes. Let the two end points of $e_{2}$ be $(a, b, c)$ and $(a, b, c+1)$. Then the two end points of $e_{1}$ are $(a-1, b+k, c)$ and

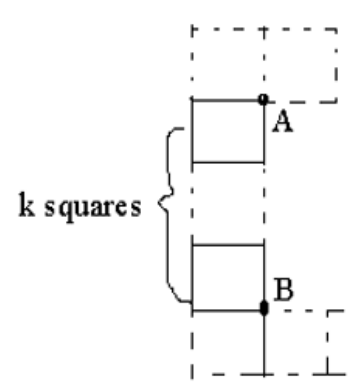

Case 1

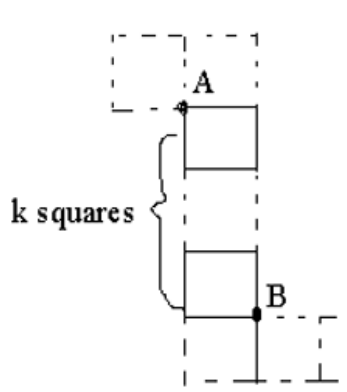

Case 2

Fig. 3. Illustration of the proof of Lemma 2 
$(a-1, b+k, c+1)$. Then the coordinates of $p_{1}$ and $p_{2}$ are $\left(a-1, b+k, c+t_{1}\right)$ and $\left(a, b, c+t_{2}\right)$ respectively, and $d_{e}\left(p_{1}, p_{2}\right)=\sqrt{\left(t_{2}-t_{1}\right)^{2}+k^{2}+1}$.

Therefore $\frac{\partial d_{e}\left(p_{1}, p_{2}\right)}{\partial t_{2}}=\frac{t_{2}-t_{1}}{\sqrt{\left(t_{2}-t_{1}\right)^{2}+k^{2}+1}}$. Let $\beta=\frac{1}{\sqrt{\left(t_{2}-t_{1}\right)^{2}+k^{2}+1}}$. This proves the lemma for Case 2.

This Lemma will be used when we prove Lemma 6] later.

Let $d_{i}=d_{e}\left(p_{i-1}, p_{i}\right)+d_{e}\left(p_{i}, p_{i+1}\right)$, where $i$ equals $1,2, \ldots$, or $m$.

Theorem 2. If $e_{i} \perp e_{j}$, where $i, j=1,2,3$ and $i \neq j$, then $e_{1}, e_{2}$ and $e_{3}$ form an end angle iff the equation $\frac{\partial\left(d_{e}\left(p_{1}, p_{2}\right)+d_{e}\left(p_{2}, p_{3}\right)\right)}{\partial t_{2}}=0$ has a unique root 0 or 1 .

Proof. Without loss of generality, we can assume that $e_{2}$ is parallel to $z$-axis.

(A) If $e_{1}, e_{2}$ and $e_{3}$ form an end angle, then by Definition 4 , the $z$-coordinates of two end points of $e_{1}$ and $e_{3}$ are equal.

Case A1. $e_{1}, e_{3}$ and the first end point of $e_{2}$ are on the same grid plane. By Lemma 1, $\frac{\partial\left(d_{e}\left(p_{1}, p_{2}\right)\right.}{\partial t_{2}}=\left(t_{2}-\alpha_{1}\right) \beta_{1}$, where $\alpha_{1}=0$ and $\beta_{1}>0$, and $\frac{\partial\left(d_{e}\left(p_{2}, p_{3}\right)\right.}{\partial t_{2}}=$ $\left(t_{2}-\alpha_{2}\right) \beta_{2}$, where $\alpha_{2}=0$ and $\beta_{2}>0$. So we have $\frac{\partial\left(d_{e}\left(p_{1}, p_{2}\right)+d_{e}\left(p_{2}, p_{3}\right)\right)}{\partial t_{2}}=$ $t_{2}\left(\beta_{1}+\beta_{2}\right)$. Therefore the equation $\frac{\partial\left(d_{e}\left(p_{1}, p_{2}\right)+d_{e}\left(p_{2}, p_{3}\right)\right)}{\partial t_{2}}=0$ has a unique root $t_{2}=0$.

Case A2. $e_{1}, e_{3}$ and the second end point of $e_{2}$ are on the same grid plane. By Lemma 1, $\frac{\partial\left(d_{e}\left(p_{1}, p_{2}\right)\right.}{\partial t_{2}}=\left(t_{2}-\alpha_{1}\right) \beta_{1}$, where $\alpha_{1}=1$ and $\beta_{1}>0$, and $\frac{\partial\left(d_{e}\left(p_{2}, p_{3}\right)\right.}{\partial t_{2}}=$ $\left(t_{2}-\alpha_{2}\right) \beta_{2}$, where $\alpha_{2}=1$ and $\beta_{2}>0$. So we have $\frac{\partial\left(d_{e}\left(p_{1}, p_{2}\right)+d_{e}\left(p_{2}, p_{3}\right)\right)}{\partial t_{2}}=$ $\left(t_{2}-1\right)\left(\beta_{1}+\beta_{2}\right)$. Therefore, equation $\frac{\partial\left(d_{e}\left(p_{1}, p_{2}\right)+d_{e}\left(p_{2}, p_{3}\right)\right)}{\partial t_{2}}=0$ has a unique root $t_{2}=1$.

(B) Conversely, if equation $\frac{\partial\left(d_{e}\left(p_{1}, p_{2}\right)+d_{e}\left(p_{2}, p_{3}\right)\right)}{\partial t_{2}}=0$ has a unique root 0 or 1 , then $e_{1}, e_{2}$ and $e_{3}$ form an end angle. Otherwise, $e_{1}, e_{2}$ and $e_{3}$ form a middle angle. By Definition 4, the $z$-coordinates of two end points of $e_{1}$ are not equal to $z$-coordinates of two end points of $e_{3}$ (Note: Without loss of generality, we can assume that $e_{2} \| z$-axis.). So $e_{1}$ and $e_{3}$ are not on the same grid plane.

Case B1. $e_{1}$ and the first end point of $e_{2}$ are on the same grid plane, while $e_{3}$ and the second end point of $e_{2}$ are on the same grid plane. By Lemma 1 $\frac{\partial\left(d_{e}\left(p_{1}, p_{2}\right)\right.}{\partial t_{2}}=\left(t_{2}-\alpha_{1}\right) \beta_{1}$, where $\alpha_{1}=0$ and $\beta_{1}>0$, while $\frac{\partial\left(d_{e}\left(p_{2}, p_{3}\right)\right.}{\partial t_{2}}=\left(t_{2}-\alpha_{2}\right) \beta_{2}$, where $\alpha_{2}=1$ and $\beta_{2}>0$. So we have $\frac{\partial\left(d_{e}\left(p_{1}, p_{2}\right)+d_{e}\left(p_{2}, p_{3}\right)\right)}{\partial t_{2}}=t_{2} \beta_{1}+\left(t_{2}-1\right) \beta_{2}$. Therefore $t_{2}=0$ or 1 is not a root of the equation $\frac{\partial\left(d_{e}\left(p_{1}, p_{2}\right)+d_{e}\left(p_{2}, p_{3}\right)\right)}{\partial t_{2}}=0$. This is a contradiction.

Case B2. $e_{1}$ and the second end point of $e_{2}$ are on the same grid plane, while $e_{3}$ and the first end point of $e_{2}$ are on the same grid plane. By Lemma 1 $\frac{\partial\left(d_{e}\left(p_{1}, p_{2}\right)\right.}{\partial t_{2}}=\left(t_{2}-\alpha_{1}\right) \beta_{1}$, where $\alpha_{1}=1$ and $\beta_{1}>0$, while $\frac{\partial\left(d_{e}\left(p_{2}, p_{3}\right)\right.}{\partial t_{2}}=\left(t_{2}-\alpha_{2}\right) \beta_{2}$, where $\alpha_{2}=0$ and $\beta_{2}>0$. So we have $\frac{\partial\left(d_{e}\left(p_{1}, p_{2}\right)+d_{e}\left(p_{2}, p_{3}\right)\right)}{\partial t_{2}}=\left(t_{2}-1\right) \beta_{1}+t_{2} \beta_{2}$. Therefore, $t_{2}=0$ or 1 is not a root of the equation $\frac{\partial\left(d_{e}\left(p_{1}, p_{2}\right)+d_{e}\left(p_{2}, p_{3}\right)\right)}{\partial t_{2}}=0$. This is a contradiction as well. 
Theorem 3. If $e_{i} \perp e_{j}$, where $i, j=1,2,3$ and $i \neq j$, then $e_{1}, e_{2}$ and $e_{3}$ form a middle angle iff the equation $\frac{\partial\left(d_{e}\left(p_{1}, p_{2}\right)+d_{e}\left(p_{2}, p_{3}\right)\right)}{\partial t_{2}}=0$ has a root $t_{2_{0}}$ such that $0<t_{2_{0}}<1$.

Proof. If $e_{1}, e_{2}$ and $e_{3}$ form a middle angle, then by Definition $4, e_{1}, e_{2}$ and $e_{3}$ do not form an end angle. By Theorem 2, 0 or 1 is not a root of the equation $\frac{\partial\left(d_{e}\left(p_{1}, p_{2}\right)+d_{e}\left(p_{2}, p_{3}\right)\right)}{\partial t_{2}}=0$. By Lemma 1 $\frac{\partial\left(d_{e}\left(p_{1}, p_{2}\right)+d_{e}\left(p_{2}, p_{3}\right)\right)}{\partial t_{2}}=\left(t_{2}-\alpha_{1}\right) \beta_{1}+\left(t_{2}-\right.$ $\left.\alpha_{2}\right) \beta_{2}$, where $\alpha_{1}, \alpha_{2}$ are 0 or $1, \beta_{1}>0$ is a function of $t_{1}$ and $t_{2}$, and $\beta_{2}>0$ is a function of $t_{2}$ and $t_{3}$. So $\alpha_{1} \neq \alpha_{2}$. (i.e., $\alpha_{1}=0$ and $\alpha_{2}=1$ or $\alpha_{1}=1$ and $\left.\alpha_{2}=0\right)$. Therefore the equation $\frac{\partial\left(d_{e}\left(p_{1}, p_{2}\right)+d_{e}\left(p_{2}, p_{3}\right)\right)}{\partial t_{2}}=0$ has a root $t_{2_{0}}$ such that $0<t_{2_{0}}<1$.

Conversely, if the equation $\frac{\partial\left(d_{e}\left(p_{1}, p_{2}\right)+d_{e}\left(p_{2}, p_{3}\right)\right)}{\partial t_{2}}=0$ has a root $t_{2_{0}}$ such that $0<t_{2_{0}}<1$, then by Theorem 2, $e_{1}, e_{2}$ and $e_{3}$ do not form an end angle. By Definition 4, $e_{1}, e_{2}$ and $e_{3}$ do form a middle angle.

Assume that $e_{0} \perp e_{1}, e_{2} \perp e_{3}$, and $e_{1} \| e_{2}$. Assume that $p\left(t_{i_{0}}\right)$ is a vertex of the MLP of $g$, where $i$ equals 1 or 2 . Then we have

Lemma 3. If $e_{0}, e_{3}$ and the first end point of $e_{1}$ are on the same grid plane, and $t_{i_{0}}$ is a root of $\frac{\partial d_{i}}{\partial t_{i}}=0$, then $t_{i_{0}}=0$, where $i$ equals 1 or 2.

Proof. From $p_{0}\left(t_{0}\right) p_{1}(0) \perp e_{1}$ it follows that

$$
d_{e}\left(p_{0}\left(t_{0}\right) p_{1}(0)\right)=\min \left\{d_{e}\left(p_{0}\left(t_{0}\right), p_{1}\left(t_{1}\right)\right): t_{1} \in[0,1]\right\}
$$

(see Figure 4). Analogously, we have $d_{e}\left(p_{2}(0) p_{3}\left(t_{3}\right)\right)=\min \left\{d_{e}\left(p_{2}\left(t_{2}\right), p_{3}\left(t_{3}\right)\right)\right.$ : $\left.t_{2} \in[0,1]\right\}$ and $d_{e}\left(p_{1}(0) p_{2}(0)\right)=\min \left\{d_{e}\left(p_{1}\left(t_{1}\right), p_{2}\left(t_{2}\right)\right): t_{1}, t_{2} \in[0,1]\right\}$. Therefore we have

$$
\begin{aligned}
& \min \left\{d_{e}\left(p_{0}\left(t_{0}\right), p_{1}\left(t_{1}\right)\right)+d_{e}\left(p_{1}\left(t_{1}\right), p_{2}\left(t_{2}\right)\right)+d_{e}\left(p_{2}\left(t_{2}\right), p_{3}\left(t_{3}\right)\right): t_{1}, t_{2} \in[0,1]\right\} \\
& \geq d_{e}\left(p_{0}\left(t_{0}\right), p_{1}(0)\right)+d_{e}\left(p_{1}(0), p_{2}(0)\right)+d_{e}\left(p_{2}(0), p_{3}\left(t_{3}\right)\right)
\end{aligned}
$$

Assume that we have $e_{0} \perp e_{1}, e_{m} \perp e_{m+1}$, and $e_{i} \| e_{i+1}$, (i.e., the set $\left\{e_{1}\right.$, $\left.e_{2}, \ldots, e_{m}\right\}$ is a set of maximal parallel critical edges of $g$, and $e_{0}$ or $e_{m+1}$ is an adjacent critical edge of this set). Furthermore, let $p\left(t_{i_{0}}\right)$ be a vertex of the MLP of $g$, where $i=1,2, \ldots, m-1$. Analogously, we have the following two lemmas:

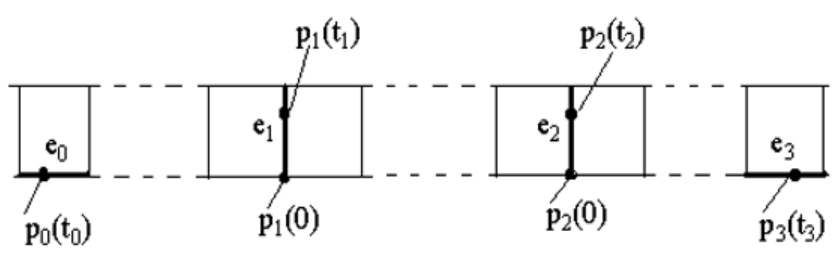

Fig. 4. Illustration of the proof of Lemma 3 
Lemma 4. If $e_{0}, e_{m+1}$ and the first point of $e_{1}$ are on the same grid plane, and $t_{i_{0}}$ is a root of $\frac{\partial d_{i}}{\partial t_{i}}=0$, then $t_{i_{0}}=0$, where $i=1,2, \ldots, m$.

Lemma 5. If $e_{0}, e_{m+1}$ and the second end point of $e_{1}$ are on the same grid plane, and $t_{i_{0}}$ is a root of $\frac{\partial d_{i}}{\partial t_{i}}=0$, then $t_{i_{0}}=1$, where $i=1,2, \ldots, m$.

Lemma 6. If $e_{0}$ and $e_{m+1}$ are on different grid planes, and $t_{i_{0}}$ is a root of $\frac{\partial d_{i}}{\partial t_{i}}=0$, where $i=1,2, \ldots, m$. Then $0<t_{1}<t_{2}<\ldots<t_{m}<1$.

Proof. Assume that $e_{0}$ and the first end point of $e_{1}$ are on the same grid plane, and $e_{m+1}$ and the second end point of $e_{1}$ are on the same grid plane. Then by Lemmas 1 and 22, $\frac{\partial d_{i}}{\partial t_{i}}$, where $i=1,2, \ldots, m$, have the following forms: $\frac{\partial d_{1}}{\partial t_{1}}=t_{1} b_{1_{1}}+\left(t_{1}-t_{2}\right) b_{1_{2}}, \frac{\partial d_{2}}{\partial t_{2}}=\left(t_{2}-t_{1}\right) b_{2_{1}}+\left(t_{2}-t_{3}\right) b_{2_{2}}, \frac{\partial d_{3}}{\partial t_{3}}=\left(t_{3}-t_{2}\right) b_{3_{1}}+$ $\left(t_{3}-t_{4}\right) b_{3_{2}}, \ldots, \frac{\partial d_{m-1}}{\partial t_{m-1}}=\left(t_{m-1}-t_{m-2}\right) b_{m-1_{1}}+\left(t_{m-1}-t_{m}\right) b_{m-1_{2}}$, and $\frac{\partial d_{m}}{\partial t_{m}}=$ $\left(t_{m}-t_{m-1}\right) b_{m_{1}}+\left(t_{m}-1\right) b_{m_{2}}$, where $b_{i_{1}}>0$, and $b_{i_{1}}$ is a function of $t_{i}$ and $t_{i-1}$, and $b_{i_{2}}>0$, and $b_{i_{2}}$ is a function of $t_{i}$ and $t_{i+1}, i=1,2, \ldots, m$.

If $t_{1_{0}}<0$, then by $\frac{\partial d_{1}}{\partial t_{1}}=0$, we have $t_{1_{0}} b_{1_{1}}+\left(t_{1_{0}}-t_{2_{0}}\right) b_{1_{2}}=0$. Since $b_{1_{1}}>0$ and $b_{1_{2}}>0$, so we have $t_{1_{0}}-t_{2_{0}}>0$, (i.e., $t_{1_{0}}>t_{2_{0}}$ ). Analogously, by $\frac{\partial d_{2}}{\partial t_{2}}=0$, so $\left(t_{2_{0}}-t_{1_{0}}\right) b_{2_{1}}+\left(t_{2_{0}}-t_{3_{0}}\right) b_{2_{2}}=0$. Then we have $t_{2_{0}}>t_{3_{0}}$. Analogously, we have $t_{3_{0}}>t_{4_{0}}, \ldots, t_{m-1_{0}}>t_{m_{0}}$. Therefore, by $\frac{\partial d_{m}}{\partial t_{m}}=\left(t_{m}-t_{m-1}\right) b_{m_{1}}+\left(t_{m}-1\right) b_{m_{2}}$, we have $t_{m_{0}}-1>0$. So we have $0>t_{1_{0}}>t_{2_{0}}>t_{3_{0}}>\ldots>t_{m_{0}}>1$. This is a contradiction.

If $t_{1_{0}}=0$, then by $\frac{\partial d_{1}}{\partial t_{1}}=0$ we have $t_{2_{0}}=0$. Analogously, by $\frac{\partial d_{2}}{\partial t_{2}}=0$ we have $t_{3_{0}}=0$. Analogously, we have $t_{4_{0}}=0, \ldots, t_{m_{0}}=0$. But, by $\frac{\partial d_{m}}{\partial t_{m}}=$ $\left(t_{m}-t_{m-1}\right) b_{m_{1}}+\left(t_{m}-1\right) b_{m_{2}}$, we have $\frac{\partial d_{m}}{\partial t_{m}}=\left(t_{m}-1\right) b_{m_{2}}=-b_{m_{2}}<0$. This is in contradiction to $\frac{\partial d_{m}}{\partial t_{m}}=0$.

If $t_{1_{0}} \geq 1$, then by $\frac{\partial d_{1}}{\partial t_{1}}=0$, we have $t_{1_{0}} b_{1_{1}}+\left(t_{1_{0}}-t_{2_{0}}\right) b_{1_{2}}=0$. Due to $b_{1_{1}}>0$ and $b_{1_{2}}>0$ we have $t_{1_{0}}-t_{2_{0}}<0$, (i.e., $t_{1_{0}}<t_{2_{0}}$ ). Analogously, by $\frac{\partial d_{2}}{\partial t_{2}}=0$ it follows that $\left(t_{2_{0}}-t_{1_{0}}\right) b_{2_{1}}+\left(t_{2_{0}}-t_{3_{0}}\right) b_{2_{2}}=0$. Then we have $t_{2_{0}}<t_{3_{0}}$. Analogously, we have $t_{3_{0}}<t_{4_{0}}, \ldots, t_{m-1_{0}}<t_{m_{0}}$. Therefore, by $\frac{\partial d_{m}}{\partial t_{m}}=\left(t_{m}-t_{m-1}\right) b_{m_{1}}+\left(t_{m}-1\right) b_{m_{2}}$, we have $t_{m_{0}}-1<0$. So we have $1 \leq t_{1_{0}}<$ $t_{2_{0}}<t_{3_{0}}<\ldots<t_{m_{0}}<1$. This is a contradiction.

Let $t_{i_{0}}$ be a root of $\frac{\partial d_{i}}{\partial t_{i}}=0$, where $i=1,2, \ldots, m$. We apply Lemmas 4, 5] and 6 and obtain

Theorem 4. $e_{0}$ and $e_{m+1}$ are on different grid plane iff $0<t_{1_{0}}<t_{2_{0}}<\ldots<$ $t_{m_{0}}<1$.

\section{An Example}

We provide one example to show that there is a simple cube-curve such that none of the vertices of its 3D MLP is a grid vertex. See Table 1, which lists the coordinates of the critical edges $e_{0}, e_{1}, \ldots, e_{19}$ of $g$. Let $v\left(t_{0}\right), v\left(t_{1}\right), \ldots, v\left(t_{19}\right)$ be 
Table 1. Coordinates of endpoints of critical edges in Figure 5

\begin{tabular}{|ccccccc|}
\hline Critical edge & $x_{i 1}$ & $y_{i 1}$ & $z_{i 1}$ & $x_{i 2}$ & $y_{i 2}$ & $z_{i 2}$ \\
\hline \hline$e_{0}$ & -1 & 4 & 7 & -1 & 4 & 8 \\
$e_{1}$ & 1 & 4 & 7 & 1 & 5 & 7 \\
$e_{2}$ & 2 & 4 & 5 & 2 & 5 & 5 \\
$e_{3}$ & 4 & 5 & 4 & 4 & 5 & 5 \\
$e_{4}$ & 4 & 7 & 4 & 5 & 7 & 4 \\
$e_{5}$ & 5 & 7 & 2 & 5 & 8 & 2 \\
$e_{6}$ & 7 & 7 & 2 & 7 & 8 & 2 \\
$e_{7}$ & 7 & 8 & 4 & 8 & 8 & 4 \\
$e_{8}$ & 8 & 10 & 4 & 8 & 10 & 5 \\
$e_{9}$ & 10 & 10 & 4 & 10 & 10 & 5 \\
$e_{10}$ & 10 & 8 & 5 & 11 & 8 & 5 \\
$e_{11}$ & 11 & 7 & 7 & 11 & 8 & 7 \\
$e_{12}$ & 12 & 7 & 7 & 12 & 7 & 8 \\
$e_{13}$ & 12 & 5 & 7 & 12 & 5 & 8 \\
$e_{14}$ & 10 & 4 & 8 & 10 & 5 & 8 \\
$e_{15}$ & 9 & 4 & 10 & 10 & 4 & 10 \\
$e_{16}$ & 9 & 0 & 10 & 10 & 0 & 10 \\
$e_{17}$ & 9 & 0 & 8 & 10 & 0 & 8 \\
$e_{18}$ & 9 & 1 & 7 & 9 & 1 & 8 \\
$e_{19}$ & -1 & 2 & 7 & -1 & 2 & 8 \\
\hline
\end{tabular}

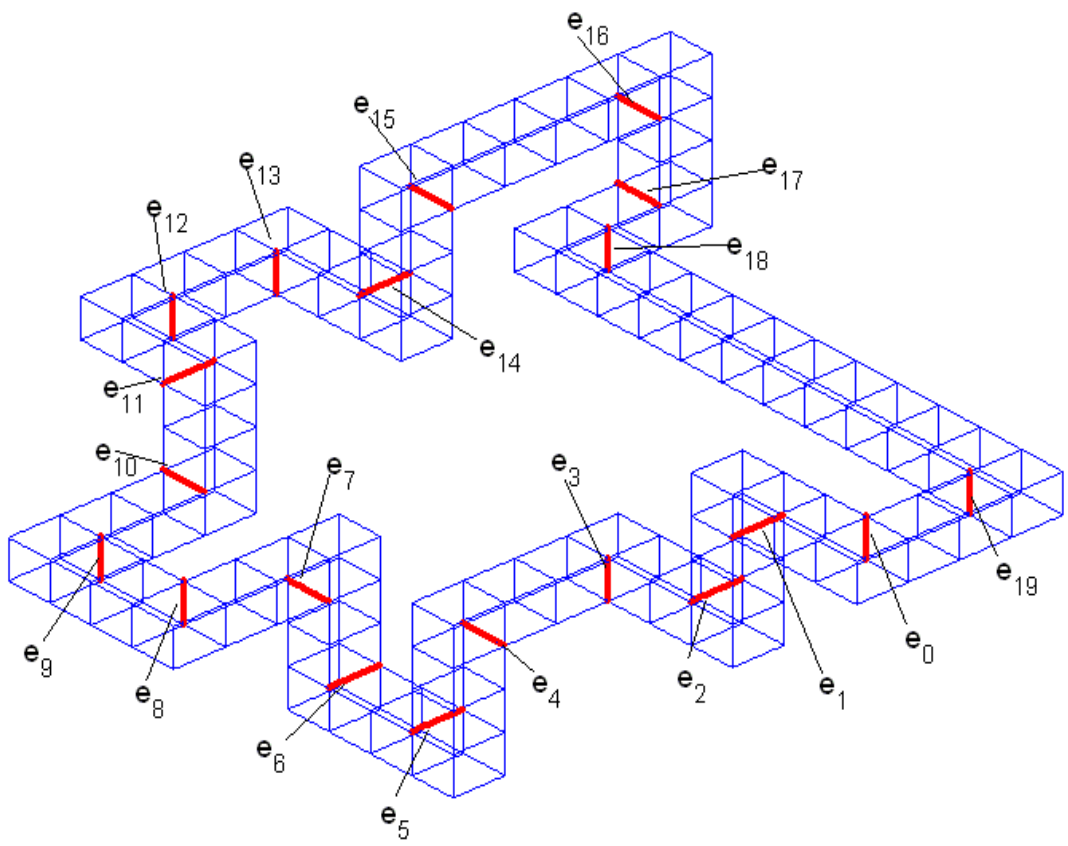

Fig. 5. A simple cube-curve such that none of the vertices of its $3 \mathrm{D}$ MLP is a grid vertex 
the vertex of the MLP of $g$ such that $v\left(t_{i}\right)$ is on $e_{i}$ and $t_{i}$ is in $[0,1]$, where $i$ $=0,1,2, \ldots, 19$. By Appendix we can see that there is not any end angle in $g$. In fact, there are 6 middle angles: $\left.\left.\left.\angle\left(e_{2}, e_{3}, e_{4}\right)\right), \angle\left(e_{3}, e_{4}, e_{5}\right)\right), \angle\left(e_{6}, e_{7}, e_{8}\right)\right)$, $\left.\left.\angle\left(e_{9}, e_{10}, e_{11}\right)\right), \angle\left(e_{10}, e_{11}, e_{12}\right)\right)$, and $\left.\angle\left(e_{13}, e_{14}, e_{15}\right)\right)$. By Theorem 3 we have $t_{3}, t_{4}, t_{7}, t_{10}, t_{11}$ and $t_{14}$ are in $(0,1)$. By Figure 5 we can see that $e_{1} \| e_{2}$ and $e_{0}$ and $e_{3}$ are on different grid planes. By Theorem 4, we have $t_{1}$ and $t_{2}$ are in $(0,1)$.

Analogously, we have $t_{5}$ and $t_{6}$ are in $(0,1) ; t_{8}$ and $t_{9}$ are in $(0,1) ; t_{12}$ and $t_{13}$ are in $(0,1) ; t_{15}, t_{16}$ and $t_{17}$ are in $(0,1)$; and $t_{18}, t_{19}$ and $t_{0}$ are in $(0,1)$. Therefore, each $t_{i}$ is in $(0,1)$, where $i=0,1, \ldots, 19$. So $g$ is a simple cube-curve such that none of the vertices of its $3 \mathrm{D}$ MLP is a grid vertex.

\section{Conclusions}

We have constructed a non-trivial simple cube-curve such that none of the vertices of its 3D MLP is a grid vertex. Indeed, by Theorems 2 and 4 and Lemmas 5 and 6. we can come to the conclusion that given a simple first class cube-curve $g$, none of the vertices of its 3D MLP is a grid point iff $g$ has not any end angle and for every set of maximal parallel edges of $g$, its two adjacent critical edges are not on the same grid plane.

It follows that the (provable correct) MLP algorithm proposed in [6] cannot be applied to this curve, because it requires at least one end angle for decomposing the curve into arcs. Of course, the rubber-band algorithm is applicable, and will produce a result (i.e., a polygonal curve). However, in this case we are still unable to show whether this result is the MLP of the given cube-curve or not.

Acknowledgements. The reviewers' comments have been very helpful for revising an earlier version of this paper.

\section{Appendix: List of $\frac{\partial d_{i}}{\partial t_{i}}(i=\mathbf{0}, \mathbf{1}, \ldots, \mathbf{1 9})$}

We compute $\frac{\partial d_{i}}{\partial t_{i}}(i=0,1, \ldots, 19)$ for $g$ as shown in Figure 5

$$
\begin{gathered}
d_{t_{0}}=\frac{t_{0}}{\sqrt{t_{0}^{2}+t_{1}^{2}+4}}+\frac{t_{0}-t_{19}}{\sqrt{\left(t_{0}-t_{19}\right)^{2}+4}} \\
d_{t_{1}}=\frac{t_{1}}{\sqrt{t_{0}^{2}+t_{1}^{2}+4}}+\frac{t_{1}-t_{2}}{\sqrt{\left(t_{1}-t_{2}\right)^{2}+5}} \\
d_{t_{2}}=\frac{t_{2}-t_{1}}{\sqrt{\left(t_{2}-t_{1}\right)^{2}+5}}+\frac{t_{2}-1}{\sqrt{\left(t_{2}-1\right)^{2}+\left(t_{3}-1\right)^{2}+4}} \\
d_{t_{3}}=\frac{t_{3}-1}{\sqrt{\left(t_{2}-1\right)^{2}+\left(t_{3}-1\right)^{2}+4}}+\frac{t_{3}}{\sqrt{t_{3}^{2}+t_{4}^{2}+4}} \\
d_{t_{4}}=\frac{t_{4}}{\sqrt{t_{3}^{2}+t_{4}^{2}+4}}+\frac{t_{4}-1}{\sqrt{\left(t_{4}-1\right)^{2}+t_{5}^{2}+4}}
\end{gathered}
$$




$$
\begin{aligned}
& d_{t_{5}}=\frac{t_{5}}{\sqrt{\left(t_{4}-1\right)^{2}+t_{5}^{2}+4}}+\frac{t_{5}-t_{6}}{\sqrt{\left(t_{5}-t_{6}\right)^{2}+4}} \\
& d_{t_{6}}=\frac{t_{6}-t_{5}}{\sqrt{\left(t_{6}-t_{5}\right)^{2}+4}}+\frac{t_{6}-1}{\sqrt{\left(t_{6}-1\right)^{2}+t_{7}^{2}+4}} \\
& d_{t_{7}}=\frac{t_{7}}{\sqrt{\left(t_{6}-1\right)^{2}+t_{7}^{2}+4}}+\frac{t_{7}-1}{\sqrt{\left(t_{7}-1\right)^{2}+t_{8}^{2}+4}} \\
& d_{t_{8}}=\frac{t_{8}}{\sqrt{\left(t_{7}-1\right)^{2}+t_{8}^{2}+4}}+\frac{t_{8}-t_{9}}{\sqrt{\left(t_{8}-t_{9}\right)^{2}+4}} \\
& d_{t_{9}}=\frac{t_{9}-t_{8}}{\sqrt{\left(t_{9}-t_{8}\right)^{2}+4}}+\frac{t_{9}-1}{\sqrt{\left(t_{9}-1\right)^{2}+t_{10}{ }^{2}+4}} \\
& d_{t_{10}}=\frac{t_{10}}{\sqrt{\left(t_{9}-1\right)^{2}+t_{10}^{2}+4}}+\frac{t_{10}-1}{\sqrt{\left(t_{10}-1\right)^{2}+\left(t_{11}-1\right)^{2}+4}} \\
& d_{t_{11}}=\frac{t_{11}-1}{\sqrt{\left(t_{11}-1\right)^{2}+\left(t_{10}-1\right)^{2}+4}}+\frac{t_{11}}{\sqrt{t_{11}^{2}+t_{12}^{2}+1}} \\
& d_{t_{12}}=\frac{t_{12}}{\sqrt{t_{11}^{2}+t_{12}^{2}+1}}+\frac{t_{12}-t_{13}}{\sqrt{\left(t_{12}-t_{13}\right)^{2}+4}} \\
& d_{t_{13}}=\frac{t_{13}-t_{12}}{\sqrt{\left(t_{13}-t_{12}\right)^{2}+4}}+\frac{t_{13}-1}{\sqrt{\left(t_{13}-1\right)^{2}+\left(t_{14}-1\right)^{2}+4}} \\
& d_{t_{14}}=\frac{t_{14}-1}{\sqrt{\left(t_{13}-1\right)^{2}+\left(t_{14}-1\right)^{2}+4}}+\frac{t_{14}}{\sqrt{t_{14}^{2}+\left(t_{15}-1\right)^{2}+4}} \\
& d_{t_{15}}=\frac{t_{15}-1}{\sqrt{t_{14}^{2}+\left(t_{15}-1\right)^{2}+4}}+\frac{t_{15}-t_{16}}{\sqrt{\left(t_{15}-t_{16}\right)^{2}+16}} \\
& d_{t_{16}}=\frac{t_{16}-t_{15}}{\sqrt{\left(t_{16}-t_{15}\right)^{2}+16}}+\frac{t_{16}-t_{17}}{\sqrt{\left(t_{16}-t_{17}\right)^{2}+4}} \\
& d_{t_{17}}=\frac{t_{17}-t_{16}}{\sqrt{\left(t_{17}-t_{16}\right)^{2}+4}}+\frac{t_{17}}{\sqrt{t_{17^{2}}+\left(t_{18}-1\right)^{2}+1}} \\
& d_{t_{18}}=\frac{t_{18}-1}{\sqrt{t_{17}{ }^{2}+\left(t_{18}-1\right)^{2}+1}}+\frac{t_{18}-t_{19}}{\sqrt{\left(t_{18}-t_{19}\right)^{2}+101}} \\
& d_{t_{19}}=\frac{t_{19}-t_{18}}{\sqrt{\left(t_{19}-t_{18}\right)^{2}+101}}+\frac{t_{19}-t_{0}}{\sqrt{\left(t_{19}-t_{0}\right)^{2}+4}}
\end{aligned}
$$




\section{References}

1. T. Bülow and R. Klette. Digital curves in 3D space and a linear-time length estimation algorithm. IEEE Trans. Pattern Analysis Machine Intelligence, 24:962-970, 2002.

2. R. Klette and A. Rosenfeld. Digital Geometry: Geometric Methods for Digital Picture Analysis. Morgan Kaufmann, San Francisco, 2004., 2004.

3. F. Sloboda, B. Zaťko, and R. Klette. On the topology of grid continua. SPIE Vision Geometry VII, 3454:52-63, 1998.

4. F. Sloboda, B. Zaťko, and J. Stoer. On approximation of planar one-dimensional grid continua. In R. Klette, A. Rosenfeld, and F. Sloboda, editors, Advances in Digital and Computational Geometry, pages 113-160. Springer, Singapore, 1998.

5. A. Jonas and N. Kiryati. Length estimation in 3-D using cube quantization, $J$. Math. Imaging and Vision, 8: 215-238, 1998.

6. F. Li and R. Klette. Minimum-length polygon of a simple cube-curve in 3D space. In Proceedings IWCIA2004, LNCS3322 (to appear).

7. J. Canny and J.H. Reif. New lower bound techniques for robot motion planning problems. Proc. IEEE Conf. Foundations Computer Science, pages 49-60, 1987.

8. J. Choi, J. Sellen, and C.-K. Yap. Approximate Euclidean shortest path in 3-space. Proc. ACM Conf. Computational Geometry, ACM Press, pages 41-48, 1994. 